\title{
Antiprotons at Solar Maximum
}

\author{
John W. Bieber, ${ }^{1}$ R. A. Burger, ${ }^{2}$ Ralph Engel, ${ }^{1}$ Thomas K. Gaisser, ${ }^{1}$ Stefan Roesler,${ }^{3}$ and Todor Stanev ${ }^{1}$ \\ ${ }^{1}$ Bartol Research Institute, University of Delaware, Newark, Delaware 19716 \\ ${ }^{2}$ Space Research Unit, School of Physics, Potchefstroom University for Christian Higher Education, \\ 2520 Potchefstroom, South Africa \\ ${ }^{3}$ CERN, CH-1211 Geneva 23, Switzerland
}

(Received 10 March 1999)

\begin{abstract}
New measurements with good statistics will make it possible to observe the time variation of cosmic antiprotons at $1 \mathrm{AU}$ through the approaching peak of solar activity. We report a new computation of the interstellar antiproton spectrum expected from collisions between cosmic protons and the interstellar gas. This spectrum is then used as input to a steady-state drift model of solar modulation, in order to provide predictions for the antiproton spectrum as well as the antiproton/proton ratio at 1 AU. Our model predicts a surprisingly large, rapid increase in the antiproton/proton ratio through the next solar maximum, followed by a large excursion in the ratio during the following decade.
\end{abstract}

PACS numbers: 98.70.Sa, 96.40.Kk

Two factors make cosmic antiprotons of special interest now. First are new experimental results [1-5], especially the abundant data from the 1997 flight of BESS [6], and the prospect of even more data from longer exposures in space $[7,8]$ and future balloon flights. Second is the opportunity to observe with good statistics the time variation of cosmic antiprotons at $1 \mathrm{AU}$ through a solar activity maximum. These observations promise to provide new insights into solar modulation, the process by which the expanding solar wind modifies the energy spectrum of cosmic rays that enter the heliosphere. At the same time, any excess of antiprotons that cannot be explained as a modulation effect may point towards a primary source of antiprotons from exotic processes [9-11].

Cosmic ray antiprotons are good probes of solar modulation. First, the main process producing antiprotons is collisions of high-energy cosmic protons with the interstellar gas. The energy spectrum of antiprotons thus produced can be computed with reasonable confidence, and we therefore have a good a priori knowledge of the input spectrum for solar modulation [11-16]. Second, the antiproton production spectrum has a distinct peak around $2 \mathrm{GeV}$ kinetic energy because of the high energy threshold for antiproton production in collisions. This is in sharp contrast [17] to the featureless monotonic spectrum of interstellar cosmic ray protons. Third, because protons and antiprotons differ only in charge sign, they are ideal for studying solar modulation effects that depend explicitly upon particle charge sign.

Existing evidence for charge sign dependent modulation appears in Fig. 1, which displays the ratio of cosmic electrons to cosmic helium observed over a 25 year period [18], together with recent observations of the electron to proton ratio made aboard Ulysses [19]. The largest variations are associated with reversals of the Sun's magnetic polarity (Fig. 1, shaded bars), which occur near the peak of solar activity. In 1970 and again in 1990, the charge ratios decreased rapidly. In 1980 the ratio jumped upwards. If the pattern continues, another large, rapid increase in the negative/positive charge ratio will occur through the polarity reversal expected in 2000 or 2001.

In the remainder of this Letter, we present a new calculation of the interstellar antiproton spectrum which we use as input to a drift model of solar modulation. We predict an interesting evolution of the $\bar{p} / p$ ratio through the upcoming solar maximum.

Interstellar antiproton spectrum. - In the framework of the standard leaky box model the continuity equation describing secondary antiproton production [14] can be written as

$$
\frac{1}{\lambda_{e}} J_{\bar{p}}\left(E_{\bar{p}}\right)+\frac{1}{\lambda_{i}} J_{\bar{p}}\left(E_{\bar{p}}\right)=\frac{c}{4 \pi\langle m\rangle} Q\left[E_{\bar{p}} ; J_{\bar{p}}\left(E_{\bar{p}}\right)\right]
$$

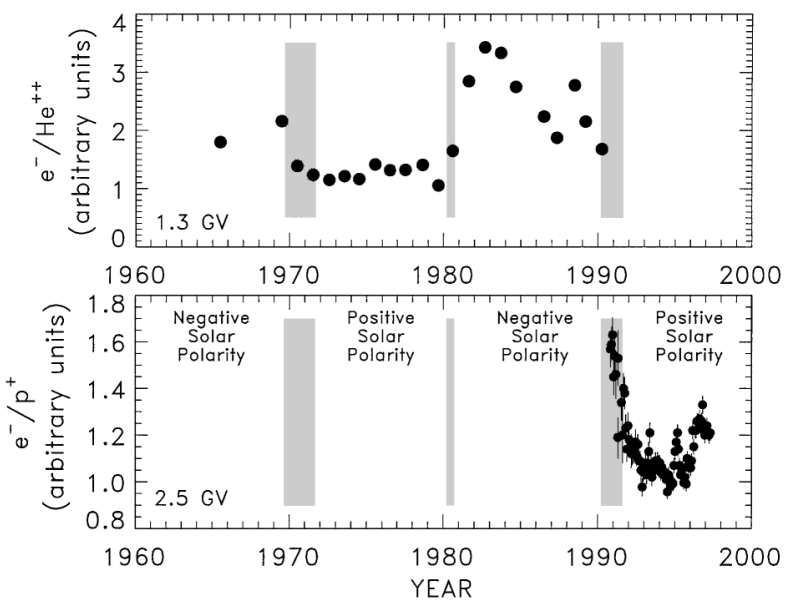

FIG. 1. Ratio of (top) cosmic electrons to cosmic helium at $1.3 \mathrm{GV}$ rigidity and (bottom) cosmic electrons to cosmic protons at $2.5 \mathrm{GV}$ rigidity. Shaded areas delimit time periods when the Sun's poloidal magnetic field was reversing. Positive and negative solar polarity refer to epochs when the magnetic field emerging from the Sun's north pole points, respectively, outward and inward. Data are from $[18,19]$. 
where $\lambda_{e}$ is the characteristic escape length, $J_{\bar{p}}\left(E_{\bar{p}}\right)$ denotes the antiproton intensity, and $\lambda_{i}$ is the interaction length for inelastic collisions of antiprotons with the interstellar gas (annihilation plus nonannihilation). The mean free path length is $\lambda_{i}\left(E_{\bar{p}}\right)=\langle m\rangle /\left\langle\sigma_{\bar{p}}^{\text {inel }}\left(E_{\bar{p}}\right)\right\rangle$, where $\langle m\rangle$ and $\left\langle\sigma_{\bar{p}}^{\text {inel }}\left(E_{\bar{p}}\right)\right\rangle$ denote the target mass and inelastic cross section averaged over the composition of the interstellar gas, respectively. The mean escape length $\lambda_{e}$ is taken from the recent fit to ratios of secondary to primary nuclei by Webber et al. [20].

The source term $Q$ is split into two parts [15] $Q\left[E_{\bar{p}} ; J_{\bar{p}}\left(E_{\bar{p}}\right)\right]=Q_{\text {prod }}\left(E_{\bar{p}}\right)+Q_{\text {scatt }}\left(E_{\bar{p}}\right)$. Here, $Q_{\text {prod }}$ is the source function for the production of antiprotons due to collisions of primary cosmic rays with the interstellar gas

$$
Q_{\mathrm{prod}}\left(E_{\bar{p}}\right)=\frac{4 \pi}{c} \sum_{i, j} n_{j} \int_{E_{\mathrm{th}}}^{\infty} \frac{2 d \sigma_{i, j \rightarrow \bar{p}}}{d E_{\bar{p}}} J_{i}\left(E_{i}\right) d E_{i},
$$

and $Q_{\text {scatt }}$ takes the inelastic scattering of antiprotons on the interstellar gas into account

$$
\begin{aligned}
Q_{\text {scatt }}\left(E_{\bar{p}}\right)= & \frac{4 \pi}{c} \sum_{j} n_{j} \\
& \times \int_{E_{\bar{p}}}^{\infty}\left\{\frac{d \sigma_{\bar{p}, j \rightarrow \bar{p}}}{d E_{\bar{p}}}+\frac{d \sigma_{\bar{p}, j \rightarrow \bar{n}}}{d E_{\bar{n}}}\right\} J_{\bar{p}}(E) d E .
\end{aligned}
$$

The index $i$ sums over primary cosmic ray particles (protons and alpha particles in our calculation) and $j$ runs over all interstellar gas target particle species $(\mathrm{H}, \mathrm{He}, \mathrm{C}, \mathrm{N}$, and $\mathrm{O})$. The particle abundances $n_{j}$ with $\sum_{j} n_{j}=1$ are taken from the data compiled in [21]. The antiproton production and inelastic scattering cross sections have been calculated with a new version of the DTUNUC Monte Carlo event generator [22,23] which uses PHOJET [24] for the simulation of elementary nucleon-nucleon collisions.

Modulation code. - The effect of gradient and curvature drifts on solar modulation has been intensively studied over the past 25 years [25-28]. Drifts in principle can provide a natural explanation for charge sign dependent modulation effects [29], because particles with opposite charge drift in opposite directions. However, in recent years there has been an emerging consensus that drifts may be important for modulation during low solar activity, but that they become unimportant for several years around solar maximum, owing to the disordered magnetic structure of the heliosphere at that time [30,31].

Recent work [32] has challenged the conventional wisdom that drifts can be ignored during high solar activity. This work finds that drifts produce a strong differentiation between modulation of positive and negative charges even during high solar activity. There may be a brief interval during the polarity reversal when the heliosphere is in a "no drift" state, but the approach to and through this state is abrupt. The observational evidence displayed in Fig. 1 favors this point of view decisively. Indeed, the largest variation of charge ratios occurs during peak solar activity in association with the polarity reversal.

The principal factors governing solar modulation are solar wind speed, tilt of the heliospheric current sheet, and the cosmic ray diffusion tensor (which also embodies the drift effect in its off-diagonal terms). For wind speed, we use a simple latitude dependent model consistent with the average properties of the solar wind [32].

The heliospheric current sheet is the surface in the solar wind that separates opposing magnetic polarities. It is essentially always tilted with respect to the solar equator, by an angle that varies from about $10^{\circ}$ at sunspot minimum to more than $70^{\circ}$ during high solar activity. The combination of solar rotation and radial expansion distorts the current sheet into a wavy "ballerina skirt" pattern, and this complex field pattern is one of the factors that leads to greater modulation during high solar activity [33].

The diffusion tensor $\mathrm{K}$ for a coordinate system with one axis parallel to the background magnetic field, $\mathbf{B}_{o}$, and the other two perpendicular to it, is

$$
\mathrm{K}=\left[\begin{array}{ccc}
\kappa_{\|} & 0 & 0 \\
0 & \kappa_{\perp, \text { polar }} & \kappa_{T} \\
0 & -\kappa_{T} & \kappa_{\perp, r \phi}
\end{array}\right]
$$

with $\kappa_{\|}$the diffusion coefficient describing the diffusion parallel to $\mathbf{B}_{o}, \kappa_{\perp, \text { polar }}$ and $\kappa_{\perp, r \phi}$ the diffusion coefficients describing the diffusion perpendicular to the background magnetic field in the polar and radial/azimuthal directions, respectively, and $\kappa_{T}$ the drift coefficient. We use quasilinear theory and a slab/two-dimensional geometry for the turbulence to calculate $\kappa_{\|}[34]$ and recent theoretical results [35] for the spatial variation of the fluctuations in the field as well as its correlation length, both quantities which appear in $\kappa_{\|}$. The perpendicular diffusion coefficients, $\kappa_{\perp, \text { polar }}$ and $\kappa_{\perp, r}$, are assumed to have the same spatial dependence as $\kappa_{\|}$, but a different rigidity $(R)$ dependence. For the drift coefficient $\kappa_{T}$ we assume that $\kappa_{T}$ /particle speed is proportional to $R^{3}$ at low rigidity, rolling over to $R^{1}$ at high rigidity. Although we believe this diffusion tensor and modulation model represent the current state-of-the-art, some limitations remain. We refer the reader elsewhere [32,36] for a full discussion.

Diffusion parameters were determined by fitting model results to proton observations at $1 \mathrm{AU}$. This procedure starts from an assumed interstellar proton spectrum. A series of recent results [37-41] indicates that the proton spectrum in the range 10 to $100 \mathrm{GeV} /$ nucleon is significantly lower than previously assumed [42]. We have therefore revised downward the standard interstellar proton spectrum [43] to fit the new data at high energy and to fit smoothly to the original result [43] at low energy, as shown in Fig. 2a. 


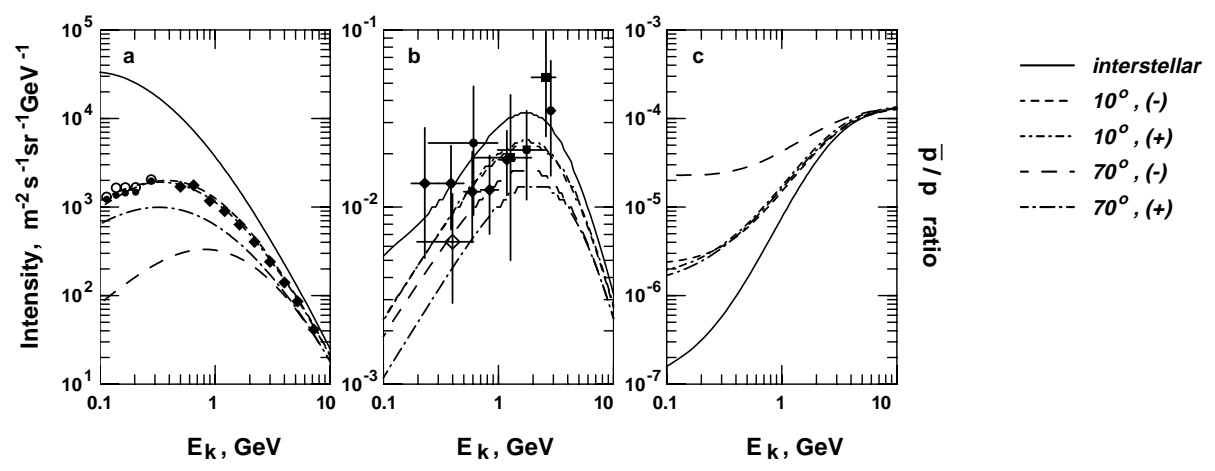

FIG. 2. Particle intensities: (a) protons, (b) antiprotons, and (c) ratio of antiproton to proton intensities. The data are taken from [37-41] for protons and from [1-4] for antiprotons.

The fit to the solar minimum proton data [44], shown in Fig. 2a, is obtained with a tilt angle of $10^{\circ}$. To model spectra near solar maximum, a tilt angle of $70^{\circ}$ is used [45] while $\kappa_{\|}, \kappa_{\perp \text {,polar }}$, and $\kappa_{\perp, r \phi}$ are all reduced by a factor of $2 / 3$. Only the sign of the magnetic field is changed when going from one solar polarity epoch to the other; i.e., it is only the direction in which particles drift that distinguishes the two polarity states in the current study.

Results. - In Fig. 2b the prediction for the local interstellar antiproton intensity is shown. Our calculation is in good agreement with the results reported in [15], with a slightly higher value below $1 \mathrm{GeV}$, and it agrees very well with the calculation of [11] at all energies. We show here only the central value of our calculation. The theoretical uncertainties due to the limited experimental data available for several quantities entering the antiproton intensity calculation will be discussed elsewhere [46], as well as the small differences with other calculations. The antiproton spectra at $1 \mathrm{AU}$ are also shown in Fig. $2 \mathrm{~b}$ with the same coding as in Fig. 2a, and Fig. 2c shows the corresponding $\bar{p} / p$ ratios.

Figure 3 displays the predicted dependence of the proton and antiproton intensity at $1 \mathrm{AU}$ (relative to interstellar level) upon tilt angle of the heliospheric current sheet, as well as the predicted dependence of their ratio. The abscissa values have been arranged so that the curves have the appearance of two successive solar cycles evolving in time. At the solar maximum of 2000 , the solar polarity switches from positive to negative. The upper panel displays a well known feature of drift models [26]: the curves for positive charges are broad during epochs of positive solar polarity (1990s), and pointy during epochs of negative polarity (decade beginning in 2000). The opposite relationship holds for negative charges.

Another difference is that protons have a greater modulation amplitude $(\sim 4 \times$ between solar maximum and solar minimum) than do antiprotons $(\sim 2 \times)$ [47]. This stems from the differing character of their input spectra: The interstellar antiproton spectrum, with its peak around $2 \mathrm{GeV}$, is "hard" compared to the proton spectrum which has many particles below $2 \mathrm{GeV}$. This feature [17] has been sug- gested [9] as a way to distinguish a component of primary antiprotons from an exotic source (such as annihilation of neutralinos in the galactic halo or evaporation from primordial black holes) from the galactic secondary component discussed in this paper. Realization of this idea relies on the primary spectum being softer than the secondary spectrum, whereas the predicted primary spectra depend strongly on the specific $\bar{p}$ production model and assumed parameters.

Summary. - Secondary galactic antiprotons provide a powerful probe of solar modulation. Protons and antiprotons have sharply different interstellar spectra. They also drift in opposite directions because of their opposite charge
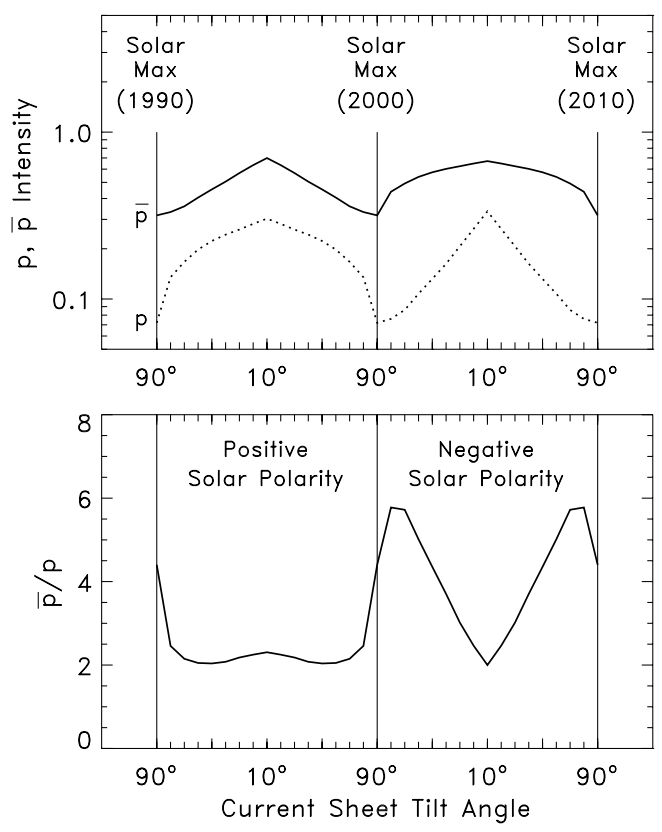

FIG. 3. Predicted dependence of (top) the proton and antiproton intensity and (bottom) the antiproton/proton ratio at $1 \mathrm{AU}$ upon the tilt angle of the heliospheric current sheet for $1 \mathrm{GeV}$ kinetic energy. Intensities are relative to interstellar level. Abscissa values are arranged so that the curves mimic the expected time variation through two solar cycles of opposite magnetic polarity (1990 to 2010). 
signs. The combination of these effects implies that the antiproton/proton ratio should display a much more interesting evolution during the next 10 years than it did during the 1990's, when the ratio was nearly constant. As we proceed through the sunspot maximum and polarity switch expected about 2000 , we predict that this ratio will rapidly increase by a factor of about 3 . Then, during the following decade, it will display a large excursion closely tied to the variation of the current sheet tilt angle. Actual observation of these variations would be a stunning validation of the importance of drift effects in solar modulation at all phases of the solar activity cycle.

This work is supported by NASA Grant No. NAG55181. J. W. B. is supported by NSF Grant No. ATM9616610 and by NASA Grant No. NAG5-7142. R. A. B. and S. R. thank the Bartol Res. Inst. for the hospitality.

[1] J. W. Mitchell et al., Phys. Rev. Lett. 76, 3057 (1996).

[2] M. Boezio et al., Astrophys. J. 487, 415 (1997).

[3] A. Moiseev et al., Astrophys. J. 474, 479 (1997).

[4] H. Matsunaga et al., Phys. Rev. Lett. 81, 4052 (1998).

[5] M. Hof et al., Astrophys. J. 467, L33 (1996).

[6] S. Orito, in Proceedings of the 29th International Conference on High Energy Physics, Vancouver, 1998 (World Scientific, Singapore, 1999), Vol. 2, p. 1463.

[7] U. Becker, in Proceedings of the 29th International Conference on High Energy Physics, Vancouver, 1998 (World Scientific, Singapore, 1999), Vol. 2, p. 1458.

[8] O. Adriani et al., in Proceedings of the 25th International Cosmic Ray Conference, Durban, 1997 (World Scientific, Singapore, 1998), Vol. 5, p. 49.

[9] T. Mitsui, K. Maki, and S. Orito, Phys. Lett. B 389, 169 (1996).

[10] A. Bottino, F. Donato, N. Fornengo, and P. Salati, Phys. Rev. D 58, 123503 (1998).

[11] L. Bergström, J. Edsjö, and P. Ullio, astro-ph/9902012.

[12] T. K. Gaisser and R. H. Maurer, Phys. Rev. Lett. 30, 1264 (1973).

[13] L. C. Tan and L. K. Ng, J. Phys. G 9, 227 (1983).

[14] T. K. Gaisser and Robert K. Schaefer, Astrophys. J. 394, 174 (1992).

[15] M. Simon, A. Molnar, and S. Roesler, Astrophys. J. 499, 250 (1998).

[16] I. V. Moskalenko and A. W. Strong, Astron. Astrophys. 338, L75 (1998).

[17] T.K. Gaisser and E.H. Levy, Phys. Rev. D 10, 1731 (1974).

[18] M. Garcia-Munoz et al., in Proceedings of the 22nd International Cosmic Ray Conference, Dublin, 1991 (Dublin Institute for Advanced Studies, Dublin, Ireland, 1991), Vol. 3, p. 497.

[19] A. Raviart et al., in Proceedings of the 25th International Cosmic Ray Conference, Durban, 1997 (World Scientific, Singapore, 1998), Vol. 2, p. 37.
[20] W. R. Webber, A. Lukasiak, F. B. McDonald, and P. Ferrando, Astrophys. J. 457, 435 (1996).

[21] J. P. Meyer, Astrophys. J. Suppl. Ser. 57, 173 (1985).

[22] A. Ferrari, J. Ranft, S. Roesler, and P. R. Sala, Z. Phys. C 71, 75 (1996).

[23] S. Roesler, R. Engel, and J. Ranft, Phys. Rev. D 57, 2889 (1998).

[24] R. Engel and J. Ranft, Phys. Rev. D 54, 4244 (1996).

[25] J. R. Jokipii, E. H. Levy, and W. B. Hubbard, Astrophys. J. 213, 861 (1977).

[26] J. Kóta and J. R. Jokipii, Astrophys. J. 265, 573 (1983).

[27] M.S. Potgieter and H. Moraal, Astrophys. J. 294, 425 (1985).

[28] W. R. Webber and M. S. Potgieter, Astrophys. J. 344, 779 (1989).

[29] M. S. Potgieter and R. A. Burger, Astron. Astrophys. 233, 598 (1990).

[30] L. J. Haasbroek, M.S. Potgieter, and J.A. Le Roux, in Proceedings of the 24th International Cosmic Ray Conference, Rome, 1995 (Argalia, Urbino, 1995), Vol. 4, p. 710.

[31] M. S. Potgieter, Space Sci. Rev. 83, 147 (1998).

[32] R. A. Burger and M. Hattingh, Astrophys. J. 505, 244 (1998).

[33] J. R. Jokipii and B. Thomas, Astrophys. J. 243, 1115 (1981).

[34] J. W. Bieber et al., Astrophys. J. 420, 294 (1994).

[35] G.P. Zank, W.H. Matthaeus, and C.W. Smith, J. Geophys. Res. 101, 17093 (1996).

[36] R. A. Burger and M. S. Potgieter (to be published).

[37] E. S. Seo et al., Astrophys. J. 378, 763 (1991).

[38] W. Menn et al., in Proceedings of the 25th International Cosmic Ray Conference, Durban, 1997 (Ref. [19]), Vol. 3, p. 409.

[39] G. Barbiellini et al., in Proceedings of the 25th International Cosmic Ray Conference, Durban, 1997 (Ref. [19]), Vol. 3, p. 369. Also, M. Boezio et al. (to be published).

[40] G. Basini et al., in Proceedings of the 25th International Cosmic Ray Conference, Durban, 1997 (Ref. [19]), Vol. 6, p. 381; see also R. Bellotti et al., Phys. Rev. D (to be published).

[41] T. Sanuki et al., in Proceedings of the Conference New Era in Neutrino Physics, Tokyo Metropolitan University, 1998 (Universal Academy Press, Tokyo, 1999), p. 123.

[42] W. R. Webber, R. L. Golden, and S. A. Stephens, in Proceedings of the 20th International Cosmic Ray Conference, Moscow, 1987 (Nauka, Moscow, 1987), Vol. 1, p. 325.

[43] W. R. Webber, Astron. Astrophys. 179, 277 (1987).

[44] J. P. L. Reinecke, H. Moraal, and F. B. McDonald, J. Geophys. Res. 98, 9417 (1993).

[45] J. A. Roux and M. S. Potgieter, Astrophys. J. 361, 275 (1990).

[46] R. Engel, T. K. Gaisser, S. Roesler, and T. Stanev (to be published).

[47] A. W. Labrador and R. A. Mewaldt, Astrophys. J. 480, 371 (1997). 